\title{
Visual Analysis of Epilepsy Diagnosis Based on Brain Functional Connections
}

\author{
Yuan Yuan', Yuying Zhu ${ }^{2 *}$, Yu He ${ }^{3}$, Yun Zhang ${ }^{3}$ \\ ${ }^{1}$ College of Computer Science and Technology, Southwest University of Science and Technology, Mianyang, China \\ ${ }^{2}$ College of Information Engineering, Southwest University of Science and Technology, Mianyang, China \\ ${ }^{3}$ Sichuan Mianyang Central Hospital, Mianyang, China \\ Email: ^zhuyuying@swust.edu.cn
}

How to cite this paper: Yuan, Y., Zhu, Y.Y., He, Y. and Zhang, Y. (2020) Visual Analysis of Epilepsy Diagnosis Based on Brain Functional Connections. Journal of Biosciences and Medicines, 8, 149-162. https://doi.org/10.4236/jbm.2020.88014

Received: July 7, 2020

Accepted: August 18, 2020

Published: August 21, 2020

Copyright $\odot 2020$ by author(s) and Scientific Research Publishing Inc. This work is licensed under the Creative Commons Attribution International License (CC BY 4.0).

http://creativecommons.org/licenses/by/4.0/

\begin{abstract}
Epilepsy is a transient neurological disorder associated with changes in the functional connections of the brain. Abnormal electrical discharges can be observed during an epileptic seizure. However, in the absence of an epileptic seizure, the anatomical structure of the brain and the electrical waves of the brain are not observed, making it difficult to explain the cause. This paper deals with together weighted imaging (DWI) sequence data in functional magnetic resonance imaging (FMRI) of epileptic patients before seizure, using Anatomical Automatic Labeling (AAL) template extracted 116 brain regions and the introduction of time series, a matrix of $116 \times 116$. Pearson correlation coefficient was calculated to investigate the pathological condition of brain function in epilepsy patients, using of neural network visualization system of innovative visual display and compared with the normal epileptic brain function to connect the image, with 38 cases of epilepsy by 187 cases of normal DWI experiment data, and can confirm the existence of brain function in patients with epilepsy connections. Cerebral neural network visualization system showed partial functional connection loss between frontal lobe and temporal lobe in epileptic group compared with normal control group.
\end{abstract}

\section{Keywords}

Primary Epilepsy, FMRI, Brain Network Connection, Edge Binding, Visualization System, Diffusion Tensor Imaging

\section{Corresponding Work}

The complex causes of epilepsy and the lack of sufficient data support make it difficult for medical experts to make a scientific and complete medical diagnosis. 
The occurrence of epilepsy is not only closely related to epileptogenic foci [1] [2], but also to the abnormal functional connection between normal brain areas and epileptogenic foci as well as the abnormal functional connection between each normal brain interval. Thus, the study of functional connectivity of brain neural network provides an extensive theoretical basis for the diagnosis and treatment of epilepsy. Resting state function MRI (rs-fMRI) has been a strong tool to study brain functional connection. So far, the diffusion tensor imaging principle is widely used in clinic, and the scan DTI sequence is added to the image post-processing to show that the tensors of different shapes of the fiber bundle satisfy the rich data dimension [3], but do not reflect the intrinsic connection of the neural network. in resting states, when the brain is not involved in any cognitive task or is not stimulated by any external output, it retains important activities that follow a clear pattern of spatial distribution [4] [5]. By processing fMRI time series images, the functional connections between different brain regions in resting state were obtained, and the functional connections of brain neural networks between patients with primary epilepsy and normal controls were compared and analyzed.

Given the maturation of noninvasive neuroimaging techniques [6], brain network analysis has become a compelling topic in data mining research, for example, the original neuroimaging data is modeled as high-order tensor [7] by introducing a time dimension to $3 \mathrm{~d}$ images. On these tensor data, some basic problems are defined [8]. Both tensor and brain networks can be trained by learning methods (tensor decomposition, feature selection) to infer relationships with specific results. Shi Lei along with other persons [9], used NodeTrix to visualize the functional network connections of the human brain, focusing on the network with clustering properties that the brain network has, following the inherent partition of the cerebral cortex, combining the traditional node link diagram with the relational matrix diagram, and showing the functional connections of the brain in blocks. Because of the complexity of brain network connectivity, visualization of brain network connectivity is unable to highlight the (ROI) characteristics of interest. Yang X. and others studied the problem of visual comparison of ROI brain networks based on block information on brain networks of interest and proposed an integrated visual analysis framework [10]. Furthermore, Cui W. and others [11] proposed a new method to construct a bundled layout of general graphs. As a layout clue of the bundle, an inner axis or skeleton of a similar edge in terms of location information is used. Combining edge clustering, distance domain and two-dimensional skeletonization, the binding layout of the general graph is gradually constructed by iteratively attracting edges to the center line of the horizontal set of its distance domain.

In the literature on functional connectivity of the brain, there are generally two research directions. One is to focus on comparing brain network differences between individuals [12]. Finn and others described how functional connectivity is unique to individuals and can be used to distinguish individuals from others [13]. Compared the similarity of subjects' correlation matrices and claimed that 
age may be a factor bringing about differences in functional connectivity between individual populations [14]. Arian and others, by analyzing the similarity between the correlation matrices of each subject [15], the effect of MRI analysis methods on individual differences was evaluated. Another direction is to analyze time-varying aspects of brain functional connectivity. Allen and others, discussed how to reveal the flexibility of functional coordination between different nervous systems [16]. Chalhoun and others, use the term "time-honney group" to represent the time-varying connectivity of the brain and review the effects of several multivariate methods on characteristic brain function. Based on complex network theory, some important topologies report the characteristics of anatomy and functional brain networks, such as small worlds, scale-free, modular, and hub regions; some new discovery has been showed up [17].

\section{Study Methods of This Paper}

In order to explore the pathology of functional connectivity of brain networks in patients with primary epilepsy, based on fMRI, this paper collected 187 normal human data and 38 images of patients with primary epilepsy, extracted areas of interest (region of interest, ROI) and calculated their Pearson product-moment correlation coefficient (also called PPMCC). The functional connectivity of brain neural networks in the experimental group was demonstrated through a visual system. Considering that the connection between functional differentiation and functional integration of brain regions is linear, PPMCC of time series is introduced. The edge binding algorithm is introduced in the visualization system to enhance the visual focus and improve the visual resolution. For the sake of highlighting the connection of neural network in brain interval, the left and right hemispheres were divided into 10 modular matrices according to the anatomical structure of the brain. The functional connections of brain network between normal control group and epilepsy group were compared by visual analysis.

\subsection{Contributions}

Joint modeling of labeled targets in data and interpretable brain networks in visualization by means of fMRI. Brain network presented in this paper is a comprehensive framework based on functional connectivity of brain neural networks. This framework achieves effectiveness from both data analysis and clinical application. Our contributions can be summarized as an empirical study of the real-world features of brain neural networks. In the absence of abnormal images fMRI patients with epilepsy, the pathological conditions of functional connectivity of brain neural networks in resting states are excavated and abnormal brain regions are displayed through a visual system. To meet the design objectives, multiple target brain regions are integrated into a selection formula, time series are introduced and Pearson correlation coefficients are calculated. In addition, it was found that the PPMCC of brain neural network connection can 
be controlled below 0.8 due to individual differences. Hence, this paper selected the threshold value at 0.8 , which mainly revealed the pathological phenomenon of functional connection of brain network in epilepsy.

Figure 1 shows the index of specific nodes of the subjects' brain network. See Appendix 1 for the specific table. Figure 2 shows the distribution of nodes and fiber connections. Figure 3 has depicted that one Example of comparison of block brain networks using NodeTrix combined with overlay design, in this brain networks, each matrix corresponds to the brain lobe in the human brain. For instance, in Figure 3, the matrix of the left half brain from top to bottom represents the left frontal lobe, left marginal lobe, left temporal lobe, left parietal lobe, left occipital lobe, respectively. Each row/column in the matrix represents a single ROI, e.g. the top two matrices are left and right frontal lobes, and each

\begin{tabular}{|c|c|c|c|c|}
\hline Mricro number Mricro named & Mriı Mricro named & Mri Mricro named & MricıMricro named & Mric Mricro named \\
\hline 1 Precentral_L & 28 Rectus_R & 55 Fusiform_L & 82 Temporal_Sup_R & 109 Vermis_1_2 \\
\hline 2 Precentral_R & 29 Insula_L & 56 Fusiform_R & 83 Temporal_Pole_Sup_L & 110 Vermis_3 \\
\hline 3 Frontal_Sup_L & 30 Insula_R & 57 Postcentral_L & 84 Temporal_Pole_Sup_R & 111 Vermis_4_5 \\
\hline 4 Frontal_Sup_R & 31 Cingulum_Ant_L & 58 Postcentral_R & 85 Temporal_Mid_L & 112 Vermis_6 \\
\hline 5 Frontal_Sup_Orb_L & 32 Cingulum_Ant_R & 59 Parietal_Sup_L & 86 Temporal_Mid_R & 113 Vermis_7 \\
\hline 6 Frontal_Sup_Orb_R & 33 Cingulum_Mid_L & 60 Parietal_Sup_R & 87 Temporal_Pole_Mid_L & 114 Vermis_8 \\
\hline 7 Frontal_Mid_L & 34 Cingulum_Mid_R & 61 Parietal_Inf_L & 88 Temporal_Pole_Mid_R & 115 Vermis_9 \\
\hline 8 Frontal_Mid_R & 35 Cingulum_Post_L & 62 Parietal_Inf_R & 89 Temporal_Inf_L & 116 Vermis_10 \\
\hline 9 Frontal_Mid_Orb_L & 36 Cingulum_Post_R & 63 SupraMarginal_L & 90 Temporal_Inf_R & \\
\hline 10 Frontal_Mid_Orb_R & 37 Hippocampus_L & 64 SupraMarginal_R & 91 Cerebelum_Crus1_L & \\
\hline 11 Frontal_Inf_Oper_L & 38 Hippocampus_R & 65 Angular_L & 92 Cerebelum_Crus1_R & \\
\hline 12 Frontal_Inf_Oper_R & 39 ParaHippocampal_L & 66 Angular_R & 93 Cerebelum_Crus2_L & \\
\hline 13 Frontal_Inf_Tri_L & 40 ParaHippocampal_R & 67 Precuneus_L & 94 Cerebelum_Crus2_R & \\
\hline 14 Frontal_Inf_Tri_R & 41 Amygdala_L & 68 Precuneus_R & 95 Cerebelum_3_L & \\
\hline 15 Frontal_Inf_Orb_L & 42 Amygdala_R & 69 Paracentral_Lobule_L & 96 Cerebelum_3_R & \\
\hline 16 Frontal_Inf_Orb_R & 43 Calcarine_L & 70 Paracentral_Lobule_R & 97 Cerebelum_4_5_L & \\
\hline 17 Rolandic_Oper_L & 44 Calcarine_R & 71 Caudate_L & 98 Cerebelum_4_5_R & \\
\hline 18 Rolandic_Oper_R & 45 Cuneus_L & 72 Caudate_R & 99 Cerebelum_6_L & \\
\hline 19 Supp_Motor_Area_L & 46 Cuneus_R & 73 Putamen_L & 100 Cerebelum_6_R & \\
\hline 20 Supp_Motor_Area_R & 47 Lingual_L & 74 Putamen_R & 101 Cerebelum_7b_L & \\
\hline 21 Olfactory_L & 48 Lingual_R & 75 Pallidum_L & 102 Cerebelum_7b_R & \\
\hline 22 Olfactory_R & 49 Occipital_Sup_L & 76 Pallidum_R & 103 Cerebelum_8_L & \\
\hline 23 Frontal_Sup_Medial_L & 50 Occipital_Sup_R & 77 Thalamus_L & 104 Cerebelum_8_R & \\
\hline 24 Frontal_Sup_Medial_R & 51 Occipital_Mid_L & 78 Thalamus_R & 105 Cerebelum_9_L & \\
\hline 25 Frontal_Mid_Orb_L & 52 Occipital_Mid_R & 79 Heschl_L & 106 Cerebelum_9_R & \\
\hline 26 Frontal_Mid_Orb_R & 53 Occipital_Inf_L & 80 Heschl_R & 107 Cerebelum_10_L & \\
\hline 27 Rectus_L & 54 Occipital_Inf_R & 81 Temporal_Sup_L & 108 Cerebelum_10_R & \\
\hline
\end{tabular}

Figure 1. Anatomical Automatic Labeling, (AAL, see Appendix 1 for the specific table).

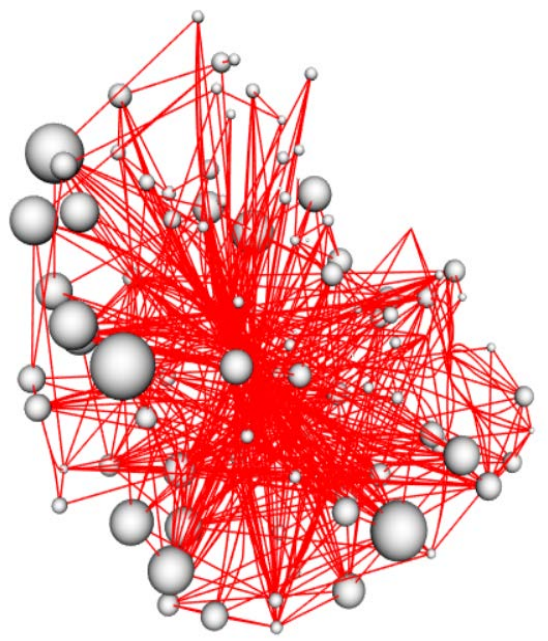

Figure 2. The nodes of a subject's brain network are located in the center of each brain region. The edges represent fiber-connected nodes, which are indexed by the label. The complete list is shown in Figure 1. 


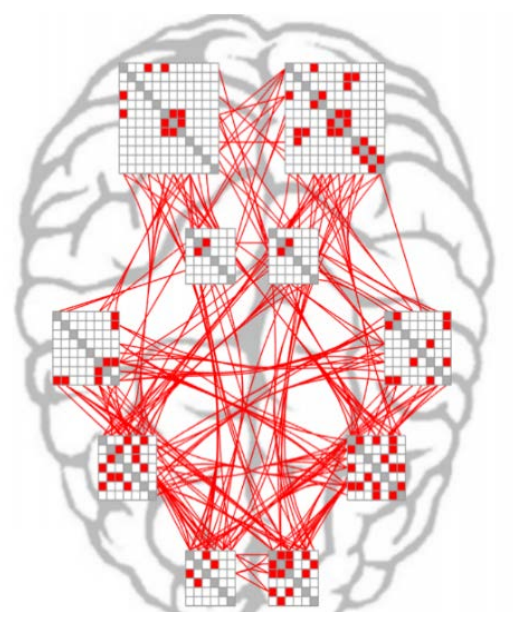

Figure 3. Subjects were tagged and the nodes of the summary brain network were grouped by regional index.

frontal lobe contains 12 of ROI. In the above tables, the 90 of ROI division reference on the templet of Anatomical Automatic Labeling (AAL) which is extensively used in Biology. Plus, the numbers and names of ROI are in accordance with templets.

In order to alleviating the visual confusion caused by the close relationship between ROI and the large number of connections, we add force-oriented edge binding algorithm to the visualization to bind these lines together. The algorithm refers to the concept of gravity and repulsion in mechanics, selects the appropriate control points for each edge, moves all the connected control points in the direction of more concentrated control points, and visually binds the edges together, thus reducing visual confusion. The force guidance algorithm of Holten and Wijk and other persons [9] are used in this paper, which is derived from the following formula.

$$
\text { OptDist }=\text { Dist }_{0} \cdot\left(\frac{C_{e}}{\min \left(C_{e}\right)}\right)^{k} \text { where } C_{e}=C_{a} \cdot C_{s} \cdot C_{p}
$$

In particular, Dist ${ }_{0}$ represents Initial distance between two control points. $C_{a}, C_{s}, C_{p}$ Represents angular compatibility, length compatibility and distance compatibility based on the two control points accordingly.

\subsection{Data Pre-Disposal}

1) Data format conversion: The raw data collected in this article are from all DICOM files, which need to be converted into NIFTI files first. 2) Removal of the first 10 point-in-time data: the images of the first 10 time nodes need to be removed before processing the image data, as there may be instability when the machine starts. 3) Time-layer correction: eliminating the time-phase difference that occurs in the interval scan so that the acquisition time for each layer in the TR is consistent. 4) Head movement correction: To prevent interference with the overall data analysis due to the writhing or sloshing of the subjects' heads, the 
images need to be corrected to approximately resting state by head movement correction. 5) Spatial standardization: To erase interference with the size of the heads of all subjects, their location in the images, and to facilitate the localization of each brain region, the brain images of all subjects need to be standardized into a standard space. 6) Nonlinear Drift: removal of the effects of linear trends (or low-frequency drift). 7) Filtering: removal of interference such as heartbeats and breathing on the images tested. Each voxel is then resampled. 8) Spatial smoothing: smoothing with a full width half-high value (FWHM) of a Gaussian kernel of 8 $\mathrm{mm} \times 8 \mathrm{~mm}$. Obtain the ROI time series of the AAL template and obtain the volume $\times 116$ time series matrix. Each column of the matrix represents the time series of each brain region. The time series of brain regions extracted from AAL template were processed by PPMCC. PPMCC between brain regions was calculated for the processed time series so that a $116 \times 116$ correlation matrix was obtained for each subject. The elements in the matrix were $r_{i j}$, representing PPMCC of node $i$ and $j$ directly. Part of the processed PPMCC data table of normal people is shown in Figure 4, and the complete data is shown in Appen$\operatorname{dix} 2$.

\subsection{PPMCC Calculation}

PPMCC between brain regions was calculated for 225 groups of test data in resting state. Each subject gets a $116 \times 116$ correlation matrix in which the elements are representing the node $i$ and $j$ direct PPMCC. Each set of data has 116 channels, each consisting of 65 time series points. The signal data of each channel as an independent signal variable, a total of 116 variables, and each channel

\begin{tabular}{|c|c|c|c|c|c|c|c|c|}
\hline 1 & 2 & 3 & 4 & 5 & 0 & 1 & 0 & 9 \\
\hline 0 & 0.496861 & 0.132462 & 0.262917 & 0.124866 & 0.152697 & 0.90486 & 0.324857 & 0.26372 \\
\hline 0.496861 & 0 & 0.794124 & 0.451357 & 0.027533 & 0.022391 & 0.445859 & 0.821638 & 0.297029 \\
\hline 0.132462 & 0.794124 & 0 & 0.658343 & 0.227276 & 0.203062 & 0.16218 & 0.635437 & 0.010836 \\
\hline 0.262917 & 0.451357 & 0.658343 & 0 & 0.488397 & 0.545625 & 0.45459 & 0.460843 & 0.142894 \\
\hline 0.124866 & 0.027533 & 0.227276 & 0.488397 & 0 & 0.890559 & 0.20993 & 0.355393 & 0.603883 \\
\hline 0.152697 & 0.022391 & 0.203062 & 0.545625 & 0.890559 & 0 & 0.192445 & 0.297532 & 0.52777 \\
\hline 0.90486 & 0.445859 & 0.16218 & 0.45459 & 0.20993 & 0.192445 & 0 & 0.409675 & 0.484182 \\
\hline 0.324857 & 0.821638 & 0.635437 & 0.460843 & 0.355393 & 0.297532 & 0.409675 & 0 & 0.560749 \\
\hline 0.26372 & 0.297029 & 0.010836 & 0.142894 & 0.603883 & 0.52777 & 0.484182 & 0.560749 & 0 \\
\hline 0.22855 & 0.015619 & 0.114519 & 0.306212 & 0.692069 & 0.689597 & 0.494465 & 0.36578 & 0.801988 \\
\hline 0.787452 & 0.375294 & 0.098462 & 0.056932 & 0.094698 & 0.019126 & 0.776154 & 0.427439 & 0.639638 \\
\hline 0.523484 & 0.292975 & 0.104941 & 0.138363 & 0.38234 & 0.327286 & 0.659143 & 0.534783 & 0.850997 \\
\hline 0.68398 & 0.429962 & 0.084135 & 0.121469 & 0.079006 & 0.029077 & 0.670023 & 0.495166 & 0.714541 \\
\hline 0.586228 & 0.359853 & 0.122329 & 0.036088 & 0.176075 & 0.106105 & 0.625357 & 0.506956 & 0.787632 \\
\hline 0.19907 & 0.634031 & 0.345064 & 0.0019 & 0.362702 & 0.192435 & 0.232789 & 0.721668 & 0.682062 \\
\hline 0.589702 & 0.087525 & 0.236777 & 0.229328 & 0.39855 & 0.412033 & 0.737261 & 0.285765 & 0.7152 \\
\hline 0.33624 & 0.348427 & 0.037662 & 0.306035 & 0.065313 & 0.074922 & 0.311875 & 0.394732 & 0.532366 \\
\hline 0.330418 & 0.137243 & 0.451751 & 0.065821 & 0.313742 & 0.310474 & 0.478103 & 0.090159 & 0.690892 \\
\hline 0.244535 & 0.847709 & 0.729745 & 0.155975 & 0.046278 & 0.140724 & 0.131248 & 0.661053 & 0.189139 \\
\hline 0.7511 & 0.272904 & 0.02819 & 0.396603 & 0.109299 & 0.109471 & 0.802114 & 0.208483 & 0.434628 \\
\hline 0.041314 & 0.038374 & 0.187769 & 0.262126 & 0.659527 & 0.609319 & 0.196707 & 0.327978 & 0.344425 \\
\hline 0.06675 & 0.014076 & 0.04752 & 0.352236 & 0.753206 & 0.772146 & 0.354396 & 0.317135 & 0.505134 \\
\hline 0.338572 & 0.646043 & 0.45468 & 0.316326 & 0.466344 & 0.302732 & 0.448625 & 0.819766 & 0.666545 \\
\hline 0.696042 & 0.219064 & 0.025129 & 0.43816 & 0.448867 & 0.414887 & 0.861969 & 0.339185 & 0.678669 \\
\hline
\end{tabular}

Figure 4. Part of the processed PPMCC data table of normal people. 
contains time series points as a set of all the data contained in each variable. Regarding these 116 signal variables, pairwise calculations result in a PPMCC matrix of scale $116 \times 116$. in this PPMCC matrix, the greater the absolute value of PPMCC, the stronger the correlation: the closer the PPMCC is to 1 or -1 , the stronger the correlation is, the closer the PPMMCC is to 0 , and the weaker the correlation is.

The calculation formulation as followed:

$$
r=\frac{N \sum x_{i} y_{i}-\sum x_{i} \sum y_{i}}{\sqrt{N \sum x_{i}^{2}-\left(\sum x_{i}\right)^{2}} \sqrt{N \sum y_{i}^{2}-\left(\sum y_{i}\right)^{2}}}
$$

\section{Virtualized System Design and Mutual Design}

\subsection{Summary}

Brain network visualization system mainly has three pages, three-dimensional brain node display, left and right juxtaposition NodeTrix contrast, superposition merge NodeTrix contrast. Set up a suspension tool box in the upper right corner of the overall page, you can choose to expand or indent. The suspension tool box is mainly used to switch three visual pages, in addition to providing setting connection strength threshold and contrast settings, normal control group and patient data are also imported through this tool box data import option.

On the 3D brain node display interface, the user can see the connection of normal people and patients as a whole for the division of AAL template brain regions. The connection strength between brain regions greater than the set threshold will show the connection lines, as showed in Figure 5(a). On the left and right side NodeTrix the contrast interface, the user can see the normal person and the patient detailed two-dimensional brain interval connection situation, as showed in Figure 5(b). The user can see the differential connections and specific numerical information between the normal and patient brain regions NodeTrix the superimposed merging contrast interface, as showed in Figure 5(c). The suspension tool box details are shown in Figure 5(d).

\subsection{Virtualized Design}

Brain network visualization system design includes two goals: one is to combine the superposition representation into the NodeTrix for visualization; the other is to combine the block hierarchy to highlight the difference contrast. Beyer and others' research has shown that node link representation and relation matrix representation are not the best choice for visual comparison. NodeTrix [18] representation using mixed representation is more suitable for visualizing block brain network connections and is more convenient for comparison between groups. Alper and others' showed that in contrast to brain functional networks, superimposed views highlight differences between groups' more than juxtaposed views. Thus, some rules have been added up into this, combining the superposition view notation on the NodeTrix, and add some interaction design. 

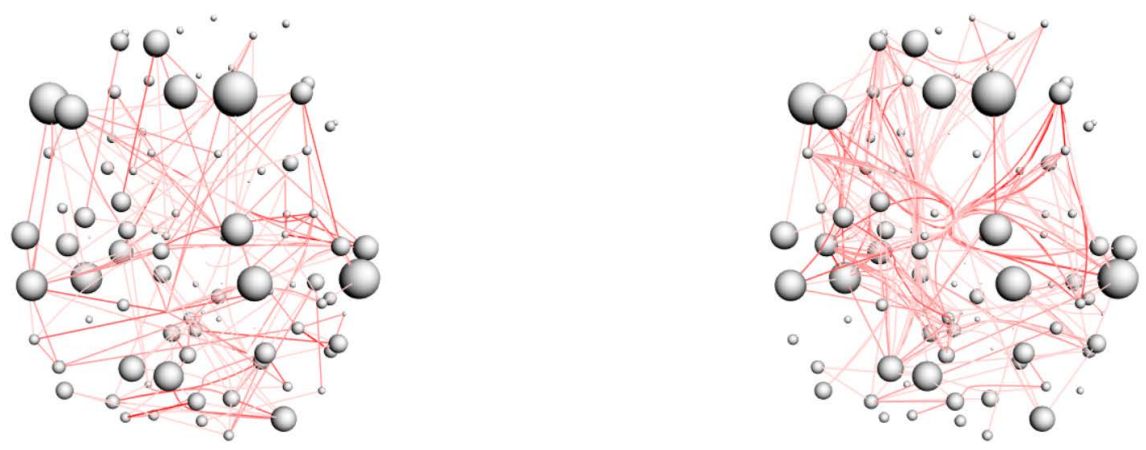

(a)
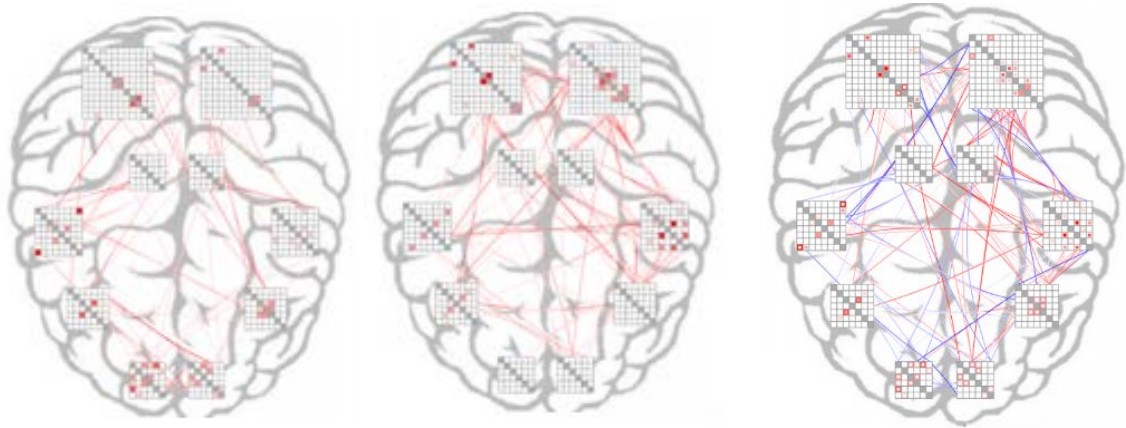

(b)

(c)

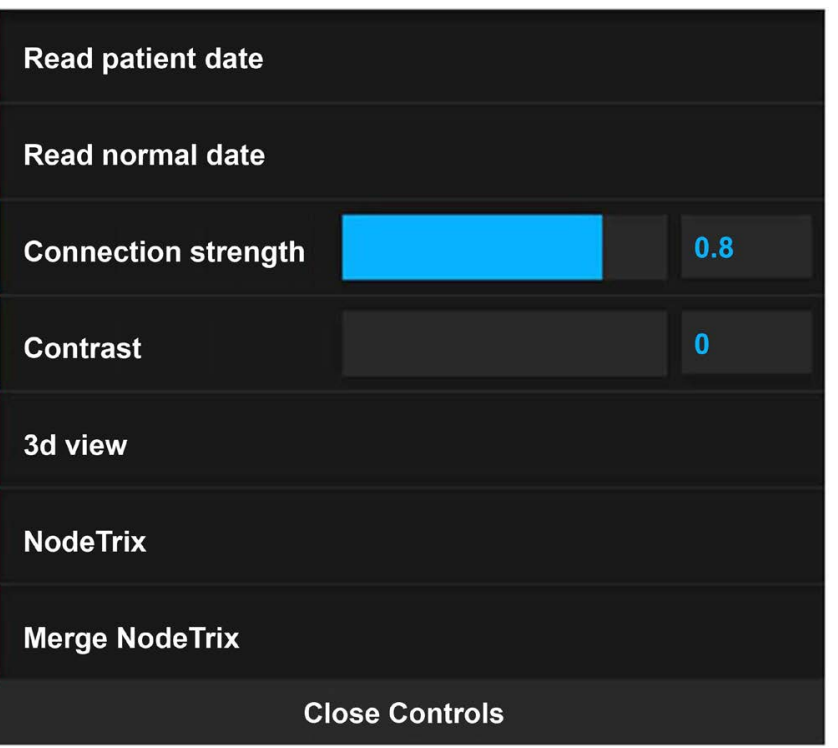

(d)

Figure 5. (a) Patient (left), normal (right), 3d brain junction display interface (upper right); (b) epilepsy group (left), normal controls (right) NodeTrix contrast figure; (c) overlay merger NodeTrix contrast interface 0 ; (d) suspension tool box.

The partitioning of 116 numbers of ROI in the figure refers to the wider application of Anatomical Automatic Labeling (AAL) templates in biology, with ROI numbers and names consistent with the templates. Large cells are mapped by patient data, and a smaller cell is nested within each relational cell, and smaller cells are mapped by data from the normal control group. Each large cell 
and his internal small cell represent the same data on the connection strength relationship between the patient and normal ROI. Inside of each matrix, the internal ROI connection is encoded with color. The saturation of color is used to represent the strength of each functional connection. The more saturated the color is and the higher the connection strength is. Besides, the meaning of different representation of small cell filling mode is also various. The specific explanation is shown in Figure 6.

\subsection{Mutual Design}

In order to reinforce the visual display effect of the system to the brain network, this paper adds some mutual design to enhance the user experience. We set up a suspension tool box through which users can switch between views. Meanwhile, the connection strength threshold and the contrast adjustment option are added, both of which range $[0,1]$. By adjusting the connection strength threshold, the connection below this set value can be shielded for easy observation and analysis. The concept of contrast refers to the normal control group connection intensity data minus the corresponding brain area patient connection intensity data. By contrast from high to low-key sections, one or more brain regions of the two groups of data can be directly displayed.

Add drag function and drop function into 3D node view, so that user can adjust the position of node view in 3D space. The user hovers over any cell or line of the brain region matrix to see the prompt box that pops up at the mouse position containing the brain region name, source ROI, target ROI and the corresponding connection strength value information, as showed in Figure 7.

\section{Case Study}

The fMRI DTI sequence of Mianyang City Central Hospital in the past three years was reviewed and analyzed. Data have collected from 74 subjects and 38

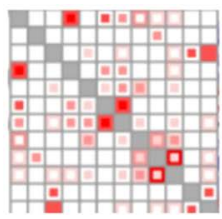

The normal control group had strong connection:

The patient has a strong connection:

The strength of the two sets of data is basically the same:

Figure 6. Matrix internal weight coding details.

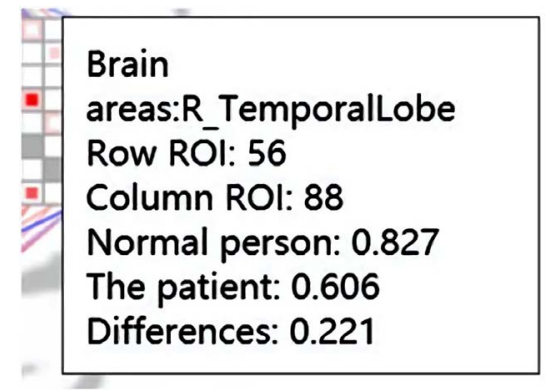

Figure 7. Block diagram of information prompt. 
patients with primary epilepsy. 74 healthy young people (average age $=26.5$ years) had no history of mental illness, neuropathy or other diseases that impaired cognitive ability, and family history. In addition, 113 normal fMRI data of Open connectome item were collected [19]. By screening the data of 187 cases in the normal control group, the PPMCC was calculated, and the functional connection map of brain neural network was obtained. The neural network connection map was extracted to show the functional connection of brain neural network in normal control group.

Experiment Group: Male, 25 years old, clinical manifestations of convulsions, disturbance of consciousness, have a history of primary epilepsy. The fMRI image is not abnormal.

Contrast group: there are 113 examples of normal people, average age is 26.5 years old, exclusive mental disease, neuropathy or other History and family history of that impair cognitive ability. The functional connections between the normal control group and the patients with primary epilepsy were compared by visualization.

As it showed in Figures 8(a)-(c) at present, Threshold strength is 0.8 .

In order to enhance visual contrast, the data of the experimental group and the control group were overlapped and processed, and the same part of the brain network was filtered out, which mainly showed the abnormal area. The red line junction section, representing the missing area of functional connectivity of the brain neural network in epileptic patients compared with the normal control group. The blue line junction part, representing the area of abnormal brain neural network connectivity in epileptic patients compared with the normal control group. The collected data of primary epilepsy group were processed by the same experimental means and the experimental results were counted. The results showed that the difference of brain neural network functional connection between frontal lobe and temporal lobe was mainly related to the loss of partial brain functional connection.

\section{Discussion}

We have used 187 normal human data to create a normal model in this paper,

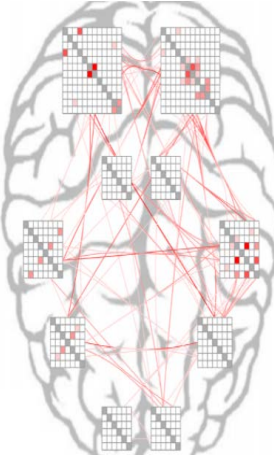

(a)

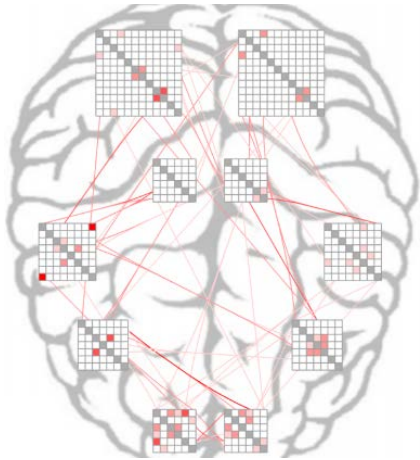

(b)

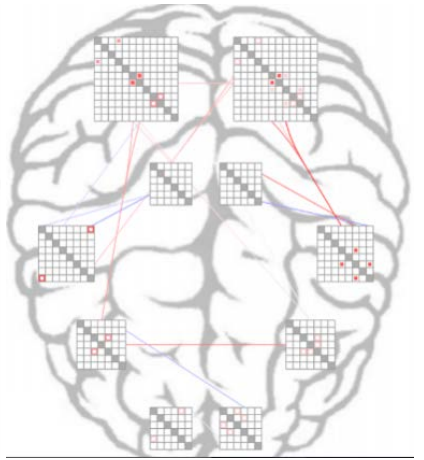

(c)

Figure 8. (a) Normal group; (b) Epilepsy patients; (c) Normal group and epileptic patients. 
because of the insufficient amount of data cannot use the deep learning method to establish a more standard normal control model. In order to avoid the influence of individual differences on the experimental results, this paper selects the experimental objects to ensure that the attributes are controllable as far as possible. In addition, in order to ensure the accuracy of the experimental results, the experimental group was divided into groups by sorting out the collected data of patients with primary epilepsy, taking into account the attributes of sex, age, education level and so on. In the course of the experiment, we were surprised to find that the effect of individual differences on functional connections of brain neural networks was minimal. In order to highlight the main pathological conditions of functional connectivity in the brain of epilepsy, we selected the threshold at 0.8 , which can ensure the filtering of the effect on the experimental results due to individual differences.

\section{Future Tendency}

We have explored the main pathological situation of functional connectivity in the brain neural network of epilepsy. The future work is to augment the amount of data, create a more accurate normal control group model, and consider various attributes within [20] to calculate the influential factors of individual differences. The pathological situation of functional connections should be more precisely highlighted in the brain neural network of epilepsy, for the interest of providing a theoretical basis for targeted treatment in clinical neurology [21].

\section{Conclusions}

In this paper, we have explored the application of the weight-connected neural network visualization method to the auxiliary diagnosis of clinical epilepsy. The visual display and analysis show the difference of brain neural network functional connectivity between epileptic patients and normal control group. The experimental results demonstrated that the difference of brain neural network functional connectivity between frontal lobe and temporal lobe was mainly relevant to the loss of partial brain functional connectivity.

The visualization system designed in this paper gives doctors a more intuitive understanding of the pathological conditions of functional connections of epileptic neural networks, and provides a valuable theoretical basis for the diagnosis and treatment of epilepsy by displaying and locating abnormal brain regions.

\section{Acknowledgements}

This work was supported by the National Defense Basic Scientific Research Program (JCKY2018404C001), and National Natural Science Foundation of China (No. 61802320).

\section{Conflicts of Interest}

The authors declare no conflicts of interest regarding the publication of this paper. 


\section{References}

[1] Widjaja, E., Li, B. and Medina, L.S. (2013) Diagnostic Evaluation in Patients with Intractable Epilepsy and Normal Findings on MRI: A Decision Analysis and Cost-Effectiveness Study. American Journal of Neuroradiology, 34, 1004-1009. https://doi.org/10.3174/ajnr.A3474

[2] Yi, C., Weina, G., Jinjian G. (2015) Seizure Outcome after Surgery in 40 Patients with Normal Preoperative MRI and Intractable Epilepsy. Sichuan Medical Journal, 36, 1403-1406.

[3] Daianu, M., Jahanshad, N., Nir, T.M., Jack, C.R., Weiner, M.W., Bernstein, M.A. and Thompson, P.M. (2015) Rich Club Analysis in the Alzheimer's Disease Connectome Reveals a Relatively Undisturbed Structural Core Network. Human Brain Mapping, 36, 3087-3103. https://doi.org/10.1002/hbm.22830

[4] Alper, B., Bach, B., Riche, N.H., et al. (2013) Weighted Graph Comparison Techniques for Brain Connectivity Analysis. Proceedings of the SIGCHI Conference on Human Factors in Computing Systems, April 2013, 483-492. https://doi.org/10.1145/2470654.2470724

[5] Wang, L. and Yu, C.S. (2008) Analytic Methods on Functional Connectivity Based on Resting-State fMRI and Their Applications. Chinese Journal of Medical Imaging Technology, 24, 1277-1280.

[6] Kong, X. and Yu, P.S. (2014) Brain Network Analysis: A Data Mining Perspective. SIGKDD Explorations, 15, 30-38. https://doi.org/10.1145/2641190.2641196

[7] Taylor, J.G. (1999) Towards the Networks of the Brain: From Brain Imaging to Consciousness. Neural Networks, 12, 943-959. https://doi.org/10.1016/S0893-6080(99)00044-1

[8] Davidson, I., Gilpin, S., Carmichael, O. and Walker, P. (2013) Net-Work Discovery via Constrained Tensor Analysis of fMRI Data. KDD, Chicago, 11-14 August 2013, 194-202. https://doi.org/10.1145/2487575.2487619

[9] Shi, L., Tong, H. and Mu, X. (2015) BrainQuest: Perception-Guided Brain Network Comparison. IEEE International Conference on Data Mining, Atlantic City, 14-17 November 2015, 379-388.

[10] Yang, X., Shi, L., Daianu, M., et al. (2016) Blockwise Human Brain Network Visual Comparison Using NodeTrix Representation. IEEE Transactions on Visualization and Computer Graphics, 23, 181-190. https://doi.org/10.1109/TVCG.2016.2598472

[11] Cui, W., Zhou, H., Qu, H., et al. (2008) Geometry-Based Edge Clustering for Graph Visualization. IEEE Transactions on Visualization and Computer Graphics, 14, 1277-1284. https://doi.org/10.1109/TVCG.2008.135

[12] Dubois, J. and Adolphs, R. (2016) Building a Science of Individual Differences from fMRI. Trends in Cognitive Sciences, 20, 425-443. https://doi.org/10.1016/j.tics.2016.03.014

[13] Geerligs, L., Rubinov, M., Henson, R.N., et al. (2015) State and Trait Components of Functional Connectivity: Individual Differences Vary with Mental State. The Journal of Neuroscience, 35, 13949-13961. https://doi.org/10.1523/JNEUROSCI.1324-15.2015

[14] Finn, E.S., Shen, X., Scheinost, D., Rosenberg, M.D., Huang, J., Chun, M.M., Papademetris, X. and Constable, R.T. (2015) Functional Connectome Fingerprinting: Identifying Individuals Using Patterns of Brain Connectivity. Nature Neuroscience, 18, 1664-1671. https://doi.org/10.1038/nn.4135

[15] Airan, R.D., Vogelstein, J.T., Pillai, J.J., Caffo, B., Pekar, J.J. and Sair, H.I. (2016) 
Factors Affecting Characterization and Localization of Interindividual Differences in Functional Connectivity Using MRI. Human Brain Mapping, 37, 1986-1997. https://doi.org/10.1002/hbm.23150

[16] Allen, E.A., Damaraju, E., Plis, S.M., Erhardt, E.B., Eichele, T. and Calhoun, V.D. (2012) Tracking Whole-Brain Connectivity Dynamics in the Resting State. Cerebral Cortex, 24, 663-676. https://doi.org/10.1093/cercor/bhs352

[17] Zhang, F.F. and Zheng, Z.G. (2012) Complex Brain Networks: Progresses and Challenges. Journal of University of Shanghai for Science and Technology, 34, 138-153.

[18] Henry, N., Fekete, J. and McGuffin, M.J. (2007) NodeTrix: A Hybrid Visualization of Social Networks. IEEE Transactions on Visualization and Computer Graphics, 13, 1302-1309. https://doi.org/10.1109/TVCG.2007.70582

[19] Open Connectome. http://openconnectomeproject.org

[20] Fujiwara, T., Chou, J.K., Mccullough, A.M., et al. (2017) A Visual Analytics System for Brain Functional Connectivity Comparison across Individuals, Groups, and Time Points. IEEE Pacific Visualization Symposium, Seoul, 18-21 April 2017, 250-259. https://doi.org/10.1109/PACIFICVIS.2017.8031601

[21] Pitkänen, A. and Lukasiuk, K. (2011) Molecular Biomarkers of Epileptogenesis. Biomarkers in Medicine, 5, 629-633. https://doi.org/10.2217/bmm.11.67 


\section{Appendix 1: The AAL Template}

\begin{tabular}{|c|c|c|c|c|c|c|c|c|c|}
\hline 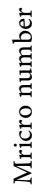 & 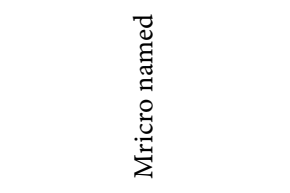 & 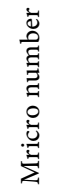 & 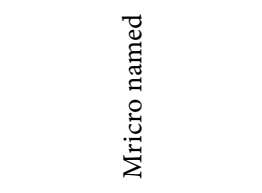 & 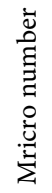 & 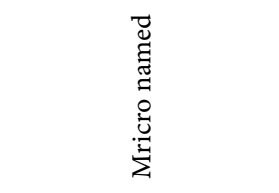 & 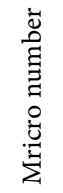 & 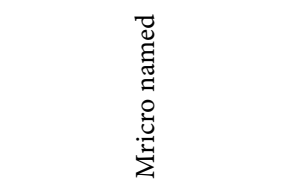 & 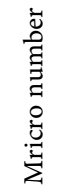 & 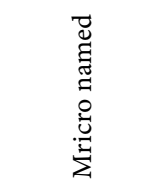 \\
\hline 1 & Precentral_L & 28 & Rectus_R & 55 & Fusiform_L & 82 & Temporal_Sup_R & 109 & Vermis_1_2 \\
\hline 2 & Precentral_R & 29 & Insula_L & 56 & Fusiform_R & 83 & Temporal_Pole_Sup_L & 110 & Vermis_3 \\
\hline 3 & Frontal_Sup_L & 30 & Insula_R & 57 & Postcentral_L & 84 & Temporal_Pole_Sup_R & 111 & Vermis_4_5 \\
\hline 4 & Frontal_Sup_R & 31 & Cingulum_Ant_L & 58 & Postcentral_R & 85 & Temporal_Mid_L & 112 & Vermis_6 \\
\hline 5 & Frontal_Sup_Orb_L & 32 & Cingulum_Ant_R & 59 & Parietal_Sup_L & 86 & Temporal_Mid_R & 113 & Vermis_7 \\
\hline 6 & Frontal_Sup_Orb_R & 33 & Cingulum_Mid_L & 60 & Parietal_Sup_R & 87 & Temporal_Pole_Mid_L & 114 & Vermis_8 \\
\hline 7 & Frontal_Mid_L & 34 & Cingulum_Mid_R & 61 & Parietal_Inf_L & 88 & Temporal_Pole_Mid_R & 115 & Vermis_9 \\
\hline 8 & Frontal_Mid_R & 35 & Cingulum_Post_L & 62 & Parietal_Inf_R & 89 & Temporal_Inf_L & 116 & Vermis_10 \\
\hline 9 & Frontal_Mid_Orb_L & 36 & Cingulum_Post_R & 63 & SupraMarginal_L & 90 & Temporal_Inf_R & & \\
\hline 10 & Frontal_Mid_Orb_R & 37 & Hippocampus_L & 64 & SupraMarginal_R & 91 & Cerebelum_Crus1_L & & \\
\hline 11 & Frontal_Inf_Oper_L & 38 & Hippocampus_R & 65 & Angular_L & 92 & Cerebelum_Crus1_R & & \\
\hline 12 & Frontal_Inf_Oper_R & 39 & ParaHippocampal_L & 66 & Angular_R & 93 & Cerebelum_Crus2_L & & \\
\hline 13 & Frontal_Inf_Tri_L & 40 & ParaHippocampal_R & 67 & Precuneus_L & 94 & Cerebelum_Crus2_R & & \\
\hline 14 & Frontal_Inf_Tri_R & 41 & Amygdala_L & 68 & Precuneus_R & 95 & Cerebelum_3_L & & \\
\hline 15 & Frontal_Inf_Orb_L & 42 & Amygdala_R & 69 & Paracentral_Lobule_L & 96 & Cerebelum_3_R & & \\
\hline 16 & Frontal_Inf_Orb_R & 43 & Calcarine_L & 70 & Paracentral_Lobule_R & 97 & Cerebelum_4_5_L & & \\
\hline 17 & Rolandic_Oper_L & 44 & Calcarine_R & 71 & Caudate_L & 98 & Cerebelum_4_5_R & & \\
\hline 18 & Rolandic_Oper_R & 45 & Cuneus_L & 72 & Caudate_R & 99 & Cerebelum_6_L & & \\
\hline 19 & Supp_Motor_Area_L & 46 & Cuneus_R & 73 & Putamen_L & 100 & Cerebelum_6_R & & \\
\hline 20 & Supp_Motor_Area_R & 47 & Lingual_L & 74 & Putamen_R & 101 & Cerebelum_7b_L & & \\
\hline 21 & Olfactory_L & 48 & Lingual_R & 75 & Pallidum_L & 102 & Cerebelum_7b_R & & \\
\hline 22 & Olfactory_R & 49 & Occipital_Sup_L & 76 & Pallidum_R & 103 & Cerebelum_8_L & & \\
\hline 23 & Frontal_Sup_Medial_L & 50 & Occipital_Sup_R & 77 & Thalamus_L & 104 & Cerebelum_8_R & & \\
\hline 24 & Frontal_Sup_Medial_R & 51 & Occipital_Mid_L & 78 & Thalamus_R & 105 & Cerebelum_9_L & & \\
\hline 25 & Frontal_Mid_Orb_L & 52 & Occipital_Mid_R & 79 & Heschl_L & 106 & Cerebelum_9_R & & \\
\hline 26 & Frontal_Mid_Orb_R & 53 & Occipital_Inf_L & 80 & Heschl_R & 107 & Cerebelum_10_L & & \\
\hline 27 & Rectus_L & 54 & Occipital_Inf_R & 81 & Temporal_Sup_L & 108 & Cerebelum_10_R & & \\
\hline
\end{tabular}

\section{Appendix 2: PPMCC Results for a Normal Person}

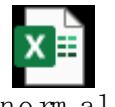

norm al

p e rso n.x lsx 